and/or perianal skin. The lesions are locally invasive and difficult to manage because of the voluminous tissue bulk and recurrence potential. Underlying immune defect in the background of HIV infection may enhance tumour-aggressiveness in these lesions. Three HIV-infected patients with BLT have been reported.

Case reports Patient 1: A 46-year-old woman presented with a cauliflower-like pink, fleshy mass of 18 months duration, protruding through the anal orifice, causing discomfort and disturbance in defecation. She was HIV-infected, acquired through conjugal relation. In addition to the above lesion, she had multiple genital molluscum contagiosum and bowenoid papulosis. Her CD4 T cell count was 350 and she was not on antiretroviral therapy (ART). Histopathology of the lesion was suggestive of BLT. The patient was treated with cryotherapy followed by surgical debulking of the lesion. ART was advised. Patient 2: A 35-year-old commercial sex worker presented with a large, variegated growth involving labia minora, extending to perineum, perianal region and lower part of gluteal folds, since last 5 years. There was sudden increase in extent and ulceration of the lesion since last 6 months. In the pubic region she had a conglomerated lesion of multiple condyloma acuminata and multiple bowenoid papulosis. She was HIV-infected with CD4 T cell count of 660. The lesion was biopsied from multiple sites and histopathological examination was consistent with BLT with grade II squamous cell carcinoma in some specimens. The patient was treated with surgical debulking followed by radiotherapy. Patient 3: A 40-year-old man with history of high risk sexual practices and intravenous drug abuse presented with multiple, fleshy masses over penis, scrotum and peno-scrotal junction, giving rise to deformed appearance of the genitalia. The lesions were present since several years with slow growth. He was HIV positive with a CD4 T cell count of 300 . He was on ART since last 6 months. Histopathology of the lesion was suggestive of BLT. The patient was treated with cryotherapy followed by surgery.

Conclusion HIV-infected patients with Buschke Löwenstein tumour are at a higher risk of developing invasive squamous cell carcinoma because of higher incidence of abnormal anal and genital cytology, presence of HPV-DNA in these cells and a low CD4 T cell count.

\section{P3-S3.04 CLINICAL SCREENING OF SEXUALLY TRANSMITTED INFECTIONS AND RISK FACTORS AMING PEOPLE LIVING WITH HIV AT THE UNIVERSITY HOSPITAL OF COTONOU}

doi:10.1136/sextrans-2011-050108.453

${ }^{1} \mathrm{C}$ Ahouada, ${ }^{1} \mathrm{~J}$ Bashi, ${ }^{2} \mathrm{M}$ D Zannou, ${ }^{1} \mathrm{~A}$ Gougounon, ${ }^{1} \mathrm{~J}$ Akakpo, ${ }^{1} \mathrm{R}$ Zinsou, ${ }^{1} \mathrm{C}$ d'Almeida, ${ }^{1} \mathrm{C}$ Ahomadegbe, ${ }^{2} \mathrm{~A}$ Azon-kouanou, ${ }^{2} \mathrm{G}$ Ade, ${ }^{2} \mathrm{~F}$ Houngbe. ${ }^{1}$ Amulatory treatment center of academyc hospital, cotonou, Benin; ${ }^{2}$ Department of internal medicine, Benin

Background Many studies have shown an association between the presence of a sexually transmitted infection (STI) and the occurrence of HIV infection. Because of improved prognosis and quality of life due to antiretroviral (ARV), people living with HIV (PLHIV) tend to abandon safer sex practices. The consequence could be the resurgence of sexually transmitted infections. The objective of this study is to determine the prevalence of STIs and associated factors in the population of PLHIV in order to lay appropriate prevention strategies.

Methods This is a descriptive cross-sectional study conducted from January to February 2011 on PLHIV admitted in consultation at Centre de Traitement ambulatoire, of academic Hospital of Cotonou. An interrogation and urogenital examination were realised systematically by physicians in all patients. STI diagnosis was presumptive, based on observed lesions. The dependent variable studied is the presence of suggestive lesions of STIs. The data entry was made using Epi Data 3.1 and statistical analysis with SAS version 9.2. Proportions were compared using the $\chi^{2}$ test. The significance level was $5 \%$ and CIs at $95 \%$.

Results 85 patients were recruited, 27 (31.76\%) had suggestive lesions of STIs. The STIs lesions found in genitals were: irritation $(21.18 \%)$, papular lesions $(12.94 \%)$, discharge $(10.59 \%)$, ulcers $(9.41 \%)$, and malodorous vaginal discharge $(4.71 \%)$. The presumptive diagnosis raised in front of these lesions were: genital candidiasis $(23.53 \%)$, genital herpes (8.24\%), condyloma (3.53\%), Chlamydia $(2.35 \%)$, syphilis $(1.18 \%)$. STI prevalence was $25 \%$ in patients on ARVs as against $52 \%$ among untreated $\mathrm{OR} 0.30$ [0.10 to 0.84$], \mathrm{p}=0.02$. The median CD4 count at diagnosis was 379 cells/ $\mu$ l. The majority of patients, that to say $58 \%$, continues to maintain unprotected sex. They were $24 \%$ reported having procreated after knowledge of their positive HIV status. This indicates a risk of sexual transmission of infections.

Conclusion STIs are frequent in people with HIV even in patients treated with ARVs. Risk behaviours of STIs and HIV transmission are particularly observed in patients whose clinical and immunological status become satisfying. It appears important to emphasise the observance of security measures in monitoring of patients with HIV, especially when their clinical and immunological status are being improved.

\section{P3-S3.05 COTRIMOXAZOLE RESISTANT RESPIRATORY OPPORTUNISTIC PATHOGENS IN HIV AND AIDS PATIENTS IN LAGOS, NIGERIA}

doi:10.1136/sextrans-2011-050108.454

N Idika, M Anidobe, D Onwujekwe, 0 Idigbe. Nigerian Institute of Medical Research, Lagos, Nigeria

Introduction Opportunistic infections, viral, bacterial, fungal and parasitic are the most common cause of morbidity and mortality in HIV patients. Of the different antibiotics used for bacterial respiratory tract infections, cotrimoxazole appears to be a drug of choice in most developing countries because of its very low cost. Studies in Ivory Coast showed that cotrimoxazole decreased hospitalisation in $50 \%$ of all HIV patients and decreased mortality by $50 \%$ when given to HIV positive TB patients. Recently Streptococus pneumoniae, Haemophilus influenzae, Moraxalla catarhalis have become increasingly resistant to antibiotics and the rates vary between countries. Aware of the public health importance, the drug resistance patterns on bacterial respiratory opportunistic pathogens from HIV patients in Lagos were studied for better management of HIV in Nigeria.

Methods 310 sputum samples were collected from HIV patients presenting with respiratory complaints at ART clinics in Lagos after due informed consent from the patients and processed in the laboratory within $4 \mathrm{~h}$ using standard microbiological methods.

Results $57.1 \%$ patients were females, $70.5 \%$ of the samples grew bacterial pathogens mainly Morascella catarrholis 25.1\%, Staphylococcus aureus 9.2\%, Streptococcus pneumoniae 8.3\%, Pseudomonas aeruginosa $7.3 \%$, coaggulase negative staphylococcus 5.3\%. Some Enterobactereciae were isolated and $86.8 \%$ of isolates were susceptible to ofloxacin, $80.2 \%$ to ciprofloxacin and $12.8 \%$ to cotrimoxazole.

Conclusion Pathogens isolated were susceptible to ofloxacin, ciprofloxacin but highly resistant to cotrimoxazole, an affordable and widely used drug in African countries. This poses a challenge to management of HIV in Nigeria. 